\title{
Analysis and Development of Intelligent Tracking System for Whereabouts of the Elder

\author{
WEI Ling-ling ${ }^{a}$, YANG Wei ${ }^{b}$
}

The Center of Collaboration and Innovation, Jiangxi University of Technology, Nanchang, Jiangxi, china, 330098

arainweiling@163.com, byang_wei@163.com

Keywords: Intelligent tracking; Geo-fencing; android; Whereabouts of the elder

\begin{abstract}
Duo to occurrence missing frequently of the elder, it is designing a tracking system with the software design tools to show the system function, it will be real time rendering the elder tracking in a dynamic map, and set up a virtual area for the elder with the geo-fencing technology, and dynamic map track the elder walk route, the virtual district manage the elder activity area, so as to better for the elder lost.
\end{abstract}

\section{Introduction}

The slogan "please care for the elder" see everywhere, However, the elder lost news frequently appear, becoming more and more serious social problems. According to survey, the lost elder main with Alzheimer's disease, and city construction and life changing is another cause of their lost. In order to prevent their lost, the people tryout many methods, such as looking for nursing care, mobile phone, wearing a ring, and so on, even locked the elder at home. These methods are played little effect, but most of them are simple contact, the specific location of the elder can not locate, and the overall effect is not ideal.

Internet of things technology [1,2] is a intelligent identification, location, tracking, monitoring and management network technology through the radio frequency identification, infrared sensors, global positioning system and other sensing devices, according to the agreement connected things with the internet, then realized information exchange and communication.

Geo-fencing is a new application of the Location Based Service, which uses a virtual fence enclose a virtual geographic border. when the phone enter or leave the given geographical area, it can receive notification and warning automatic.

This paper combined with the Internet of things technology and the geo-fencing technology, studied the intelligent tracking system of the elder, and their footprint label the map real time and form a dynamic graph, so that located the elder to prevent them lost.

\section{Requirement Analysis}

According to the market requirement, the main function requirements as below.

Location obtain real time. The system client install and run in the android phone, so that the software get the elder location real time.

Action tracking dynamic show. The software will collect the tracking data, the points drawing a figure and displaying in the map.

The geo-fencing technology application. It is studied the geo-fencing technology apply to the software, then give a virtual bound around the elder, the phone can receive notification and warning automatic when walking out the border, and the phone of family can receive the same information.

\section{The System Design}

Make most of the advantage of android technology[3,4,5] and geo-fencing technology, Analyzing and designing the tracking management system of the elder is aimed at solved the problems of the elder lost. 


\section{The System Overall Deployment Design}

Developing the software run in the android phone based on the android system, the system will send a position message to the server in set period or the server collect the data automatic, and set the virtual bound of elder action by means of geo-fencing, if them leave the domain and enter another, the system in the phone will alarming and send a position information to the server and the phone of family. At the same time, the server will drawing action tracking in map according to receive and collect data.

Specific diagram as follows:

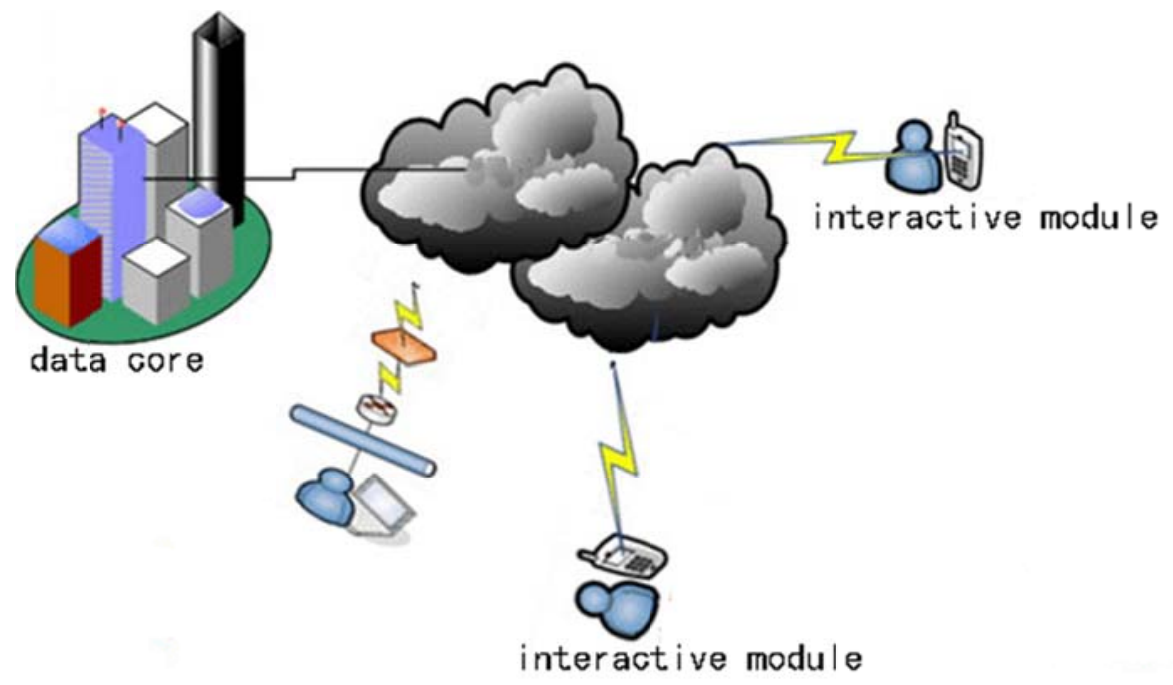

Fig.1 the overall deployment diagram

\section{The Software Design}

The system mainly has two functions, one is the dynamic acquisition of the location data of the elder and action track show, one another is managing action domain of the ellder. Below describing the overall structure of the software with the structure diagram which the name of module in frame and the lines between the square frame is express call relationship.

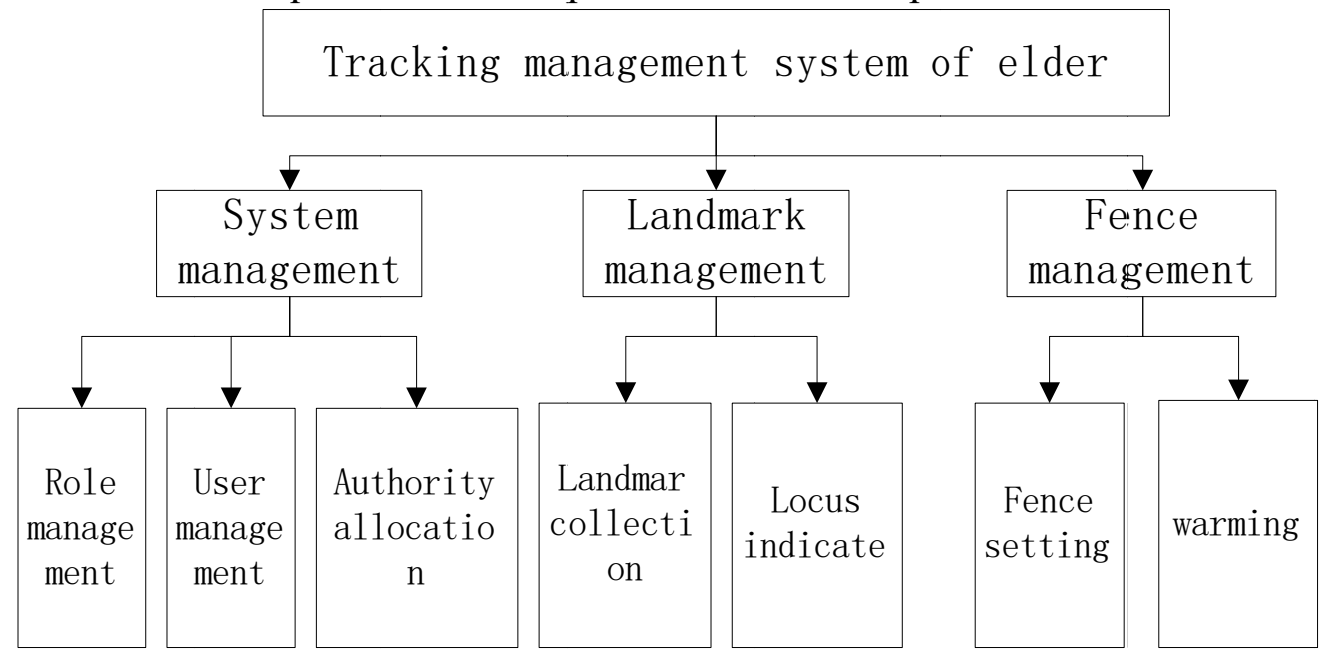

Fig.2 structure of the system module

\section{Landmark Management}

The landmark management is real time gathering the longitude and latitude of the elder, and mark in map by line and point, then forming a dynamic action curve diagram. 


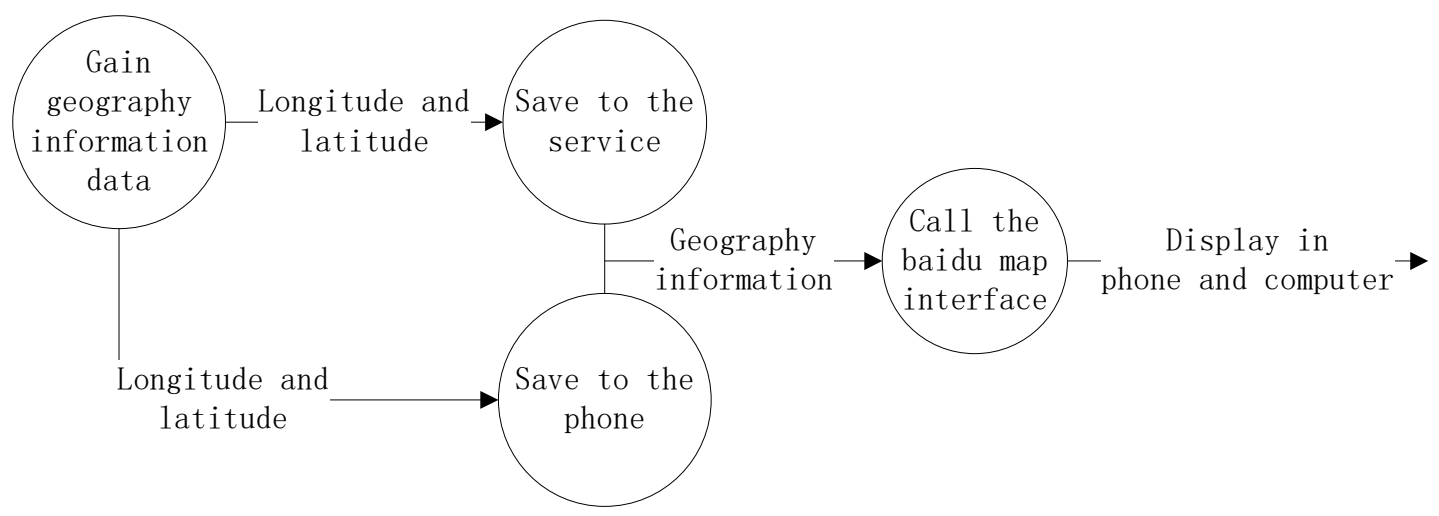

Fig.3 trail data flow diagram

\section{Fence Management}

Fence management with the help of geo-fencing, in which the geographic area was meshed. The standard of grid is in line with the business and commercial cluster of a geographic are rather than the simple match of the longitude and the latitude and city map. The family pre-establish the domain of the elder action, when the elder leave the domain, the system will warning.

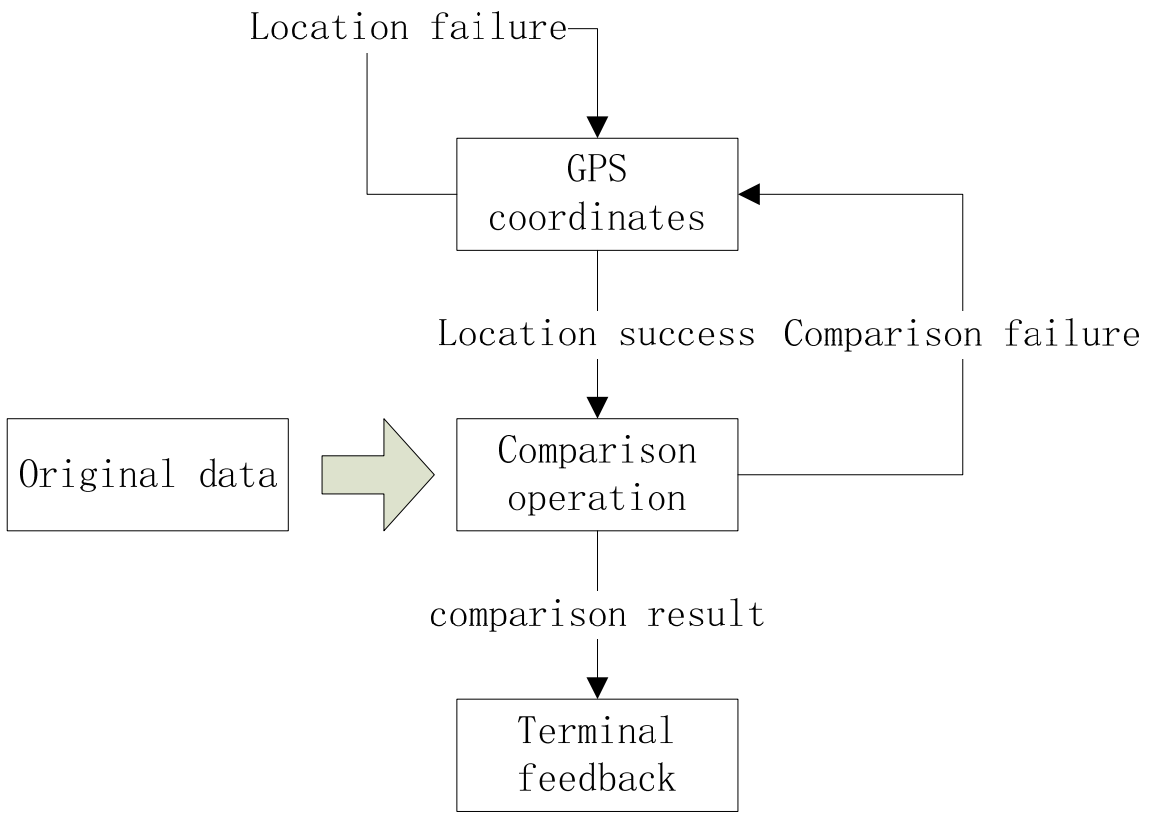

Fig.4 fence processing flow diagram

\section{Summary}

This paper combined with the internet of things technology, android technology and geo-fencing technology, studying the intelligent tracking system of the edler, and the tracking marked in map, then forming a dynamic graph. In this way, on the one hand can go to the position of the elder to prevent them loss; on the other hand the phone of the elder with the system or well-wisher can call to the family when happen the fortuitous event, then timely solving the problem. At the same time, the family can set the elder action space with the help of the geo-fencing technology, once the elder out of the bound, the phone will warning. The goal of the system is giving the elder a safe and healthy old age life. 


\section{Acknowledgement}

This work was supported by the Natural Science Foundation of school (ZR14YB04).

\section{References}

[1]yao xu dong. The comparative research on the development of internet of things technology between home and abroad[D]. Southwest jiaotong university,2012,master paper.

[2]Wu zhen yu.research on web of things service environment architecture and key technologies[D]. Beijing university of posts and telecommunications,2013,doctor paper.

[3] PAN Xiao-dong;FEI Jun. Application of Android-based Central Monitoring \& Locating System in Medical Service[J] .Chinese Medical Equipment Journal,2014,4.

[4]Mo fei.android development tutorial[M]. The posts and telecommunications press, 2010.

[5]li gang.crazy android development[M].Publishing house of electronics industry,2013. 